Methods This study was conducted in November 2019 following modified time-location sampling. Instruments included rapid tests for HIV and Hepatitis B and C and a behavioral questionnaire.

Results Our results can be grouped into three main levels: education, stigma and risky behavior. Compared with nativeborn GBM, immigrants/refugees GBM were more likely to report non-university level of education, and lower STIs knowledge. Moreover, they showed more healthcare and employment respective stigma. Hence, more heterosexual marriage, more commercial sex work, more history of syphilis in the last 12 months and a higher mean number of male sex partners in the last 6 months were noted among these communities. Positive correlations were found between STIs knowledge and socioeconomic status; feeling sufficiently informed about STIs. Being immigrants/refugees and feeling sufficiently informed about STIs were strong indicators of STIs knowledge.

Conclusion This study highlights the respective roles of education and immigrant/refugee status in STIs knowledge in Lebanon. Efforts to increase general educational opportunities for immigrants/refugees and specifically sexual education would be helpful inherently, and might also support higher STIs knowledge. Moreover, our participants most probably had to conform to cultural and religious norms and get married. This entails a degree of hiding their true identity and causing distress. Both hiding identity and experiencing stigma may push people of sexual minorities to cope in a wide range of ways and one possible strategy might be sexual risk-taking.

\section{P048 THE EMERGING IMPACT OF THE COVID-19 OUTBREAK ON SEXUAL HEALTH IN LEBANON}

I Maatouk*, M Assil, R Jaspal. Nottingham Trent University,

10.1136/sextrans-2021-sti. 192

Background The coronavirus-2019 (COVID-19) has had compounding effects on the political and economic crisis with severe economic and health consequences. Throughout the lockdown, one of the largest sexually transmitted infections (STIs) and HIV clinics in Beirut with linkages to major civil organizations in direct contact with sexual health beneficiaries has remained open for emergencies. The main objective of this study was to measure COVID19 impact on sexual health services in this clinic in Beirut.

Methods A review of electronically based STIs screenings and diagnoses for the period February-December 2020 was conducted.

Results The clinic has noted a major reduction in STI testing rates. Only 116 screenings were recorded in the mentioned period which contrasts with the 334 screenings conducted during the same period of 2019 (65\% drop in 2020). All the screenings were among men who have sex with men (MSM). On the other hand, PEP was prescribed 86 times in the period February-December 2020 compared to 67 times during the same period of time in 2019 (28\% increase). All cases of PEP prescriptions were for MSM. A range of risky behaviors and a $12 \%$ prevalence of HIV have been documented in MSM in Lebanon.

Conclusion The increase in PEP prescription for MSM indicates that sexual risk-taking has persisted despite potential fear of contracting COVID-19. Two factors could explain our
Results (1) social psychological stressors (political and economic instability, huge explosion, COVID19) may threaten identity and wellbeing, leading MSM to resort to sexual risk behaviours; (2) data on epidemiology of STIs in COVID19 time are inconsistent due to the lack of stratification by incubation period, acute versus chronic type, duration of symptoms and sexual orientation. Future studies should ascertain the impact of COVID19 on both mental and sexual health especially in stigmatized communities such as MSM.

\section{P049 PREDICTING SEXUAL RISK AND SEXUAL HEALTH SCREENING IN A SAMPLE OF UNIVERSITY STUDENTS IN LEBANON: A CROSS-SECTIONAL STUDY}

I Maatouk*, M Assi, R Jaspal. Nottingham Trent University

\subsection{6/sextrans-2021-sti.193}

Background There are very few studies from the Middle East on HIV knowledge, attitudes, and other sexual health issues among university students. This is particularly true for Lebanon. The present study focuses on factors that predict sexual risk behaviors and sexual health screening behaviors in a sample of university students in Lebanon.

Methods A diverse sample of 250 undergraduate students at a university in Beirut, Lebanon participated in a survey study. They completed measures of religiosity, ethnic identification, identity threat, psychological distress, self-harm, contraceptives usage, HIV/STI screening, and sexual risk behaviors.

Results Religion is negatively associated with a range of risky sexual behaviors. Psychological distress is associated with having sex under the influence of alcohol and drugs, engaging in a sexual activity later regretted, and feeling forced to engage in a sexual activity that they did not want to engage in. Sex under the influence of alcohol and drugs/substances, discussing methods of contraception, and willingly engaging in sexual activities later regretted had significant effects on the variance of having had condomless vaginal sex in the past month. Gender and sex under the influence of alcohol had significant effects on screening for STIs.

Conclusion Religiosity appears to be protective against identity threat and psychological distress and negatively associated with a variety of sexual risk behaviors. Perceived stigma associated with female sexuality can limit STIs screening. Interventions that focus on managing different variables (drug and alcohol use in students, unwanted pregnancy, religion, stigma related to screening, and sexual health education) are recommended.

\section{P050 MOTIVES AND BARRIERS FOR COMPLYING WITH 'SEXUAL DISTANCING' AMONG MSM DURING THE FIRST COVID-19 PANDEMIC LOCKDOWN, A QUALITATIVE STUDY}

D De Vries*, H Zimmerman, S Drückler, U Davidovich, E Hoornenborg, H de Vries. GGD Amsterdam, Amsterdam, The Netherlands

\subsection{6/sextrans-2021-sti.194}

Backgrounds On March 12,2020 the Dutch government imposed a lockdown and heath measures to curb the COVID19 pandemic. As part of social distancing, sexual distancing was one of these measures. Sexual distancing implied no sex 